\title{
Classical microscopic theory of polaritons in ionic crystals
}

\author{
A. Lerose* \\ A. Sanzeni* \\ A. Carati $^{\dagger}$ \\ L. Galgani ${ }^{\dagger}$
}

March 24, 2022

\begin{abstract}
It is well known that the optical branches of the dispersion curves of ionic crystals exhibit a polaritonic feature, i.e., a splitting about the electromagnetic dispersion line $\omega=c k$. This phenomenon is considered to be due to the retardation of the electromagnetic forces among the ions. However, the problem is usually discussed at a phenomenological level, through the introduction of a macroscopic polarization field, so that a microscopic treatment is apparently lacking. A microscopic first principles deduction is given here, in a classical frame, for a model in which the ions are dealt with as point charges. At a qualitative level it is made apparent that retardation is indeed responsible for the splitting. A quantitative comparison with the empirical data for $\mathrm{LiF}$ is also given, showing a fairly good agreement over the whole Brillouin zone.
\end{abstract}

PACS: $71.36 .+\mathrm{c}, 63.20 . \mathrm{dk}$

Keywords: Polaritons, dispersion curves, ionic crystals

${ }^{*}$ School of Physics, Università degli Studi diMilano, Italy

${ }^{\dagger}$ Dipartimento di Matematica, Via Saldini 50, Milano, I-20133, Italy, E-mail: andrea.carati@unimi.it. 


\section{Introduction}

The existence of polariton dispersion curves in ionic crystals is of great physical relevance, inasmuch as it allows one to explain the phenomenon of the dispersion of light in such crystals. Polaritonic curves exist only if one takes into account the retarded nature of the electromagnetic forces among the ions in the crystal. Indeed, if retardation is neglected one obtains the familiar Coulomb model in which the dispersion curves of the crystal lattice and those of light are completely different, whereas they coincide along the polaritonic lines. So retardation is the essential necessary ingredient in order to explain the optics of crystals, and it will be shown here that it is the retarded interaction with the far ions that is actually responsible for the splitting.

Now, a splitting of the dispersion curves of an ionic crystal about the electromagnetic line $\omega=c k$, was apparently first predicted by Born and Huang (see [1], pages 91 and 94) in the frame of a phenomenological discussion of the problem in terms of a macroscopic polarization field. Further discussions were then given by Fano [2] and Hopfield [3], still in terms of macroscopic polarization densities. The splitting was finally observed in the years 60s, first in semiconductors and later in LiF and in other ionic crystals. In conclusion, it is now commonly assumed (see for example [4], sections 7.2 and 7.3) that the phenomenon should be understood as due to retardation. However, retardation is usually introduced through the phenomenological Maxwell equations which involve, in addition to the microscopic field $\mathbf{E}$, also the macroscopic field D. A microscopic deduction is thus apparently lacking.

In the present paper such a microscopic deduction is given, in a classical frame. We consider a model in which the ions are dealt with as point particles, internal degrees of freedom being neglected. The existence of molecular repulsive forces balancing the Coulomb ones, and thus allowing for the existence of a lattice, is assumed at a phenomenological level. The Newton equations of motion for the displacements of the ions, with retardation of the electromagnetic forces taken into account, are written down in the linear approximation. At the end the model involves as free parameters the constants entering the repulsive forces, and the effective charge of each ion, in addition to the geometric parameters of the lattice and to the ions' masses. The normal modes are numerically determined for crystals with a rock salt structure, through a suitable procedure, which is required in order to take the effect of retardation into account. The three free parameters are determined by a comparison with the experimental data of the dispersion curves of $\mathrm{LiF}$, 
while the remaining parameters were taken from the literature. Finally, the electromagnetic field generated by the motions of the ions is discussed.

The main results are the following:

1. In the dispersion curves of the lattice vibrations there appear branches that are absent in the purely "mechanical or instantaneous" model (in which retardation is neglected), and correspond to the previously mentioned polaritonic splitting. The splitting turns out to be actually due to the retarded interaction with the far ions.

2. The agreement between the theoretically computed dispersion curves and the experimental ones available in the literature for the case of $\mathrm{LiF}$ (see figures 1 and 2) is, in our opinion, fairly good for all values of $\mathbf{k}$ in the whole Brillouin zone. This is obtained with no need of introducing a phenomenological value for the static dielectric constant $\varepsilon$, which is here deduced from the theory.

3. The electromagnetic field created by the motion of the ions can be decomposed into a microscopic part and a macroscopic one. The former propagates at the vacuum speed $c$, while the latter propagates according to the laws of macroscopic optics, with a phase velocity $\omega(\mathbf{k}) / k$.

4. The dispersion relation $\omega(\mathbf{k})$ of the macroscopic electric field coincides with the vibrational one of the lattice.

In Section 2 the microscopic model is described and the linearized equations of motion are obtained. In Section 3 it is shown how, due to retardation, the secular equation presents a peculiar form, which is responsible for the occurrence of the splitting. In Section 4 the dispersion curves explicitly computed for rock salt lattices are reported, and the comparison with the experimental data is performed. Some further general problems concerning the microscopic deduction of optics are discussed in Section 5. More comments are given in a conclusive section. Appendix $\mathrm{A}$ is devoted to recalling the role played by the so called Wheeler-Feynman identity[5] (which is a theorem in the present model) in ensuring the stability of the lattice. Indeed, such an identity guarantees that the microscopic dispersion relations do not contain imaginary terms, if the familiar radiation reaction force [6] acting on each ion is taken into account. Appendices $\mathrm{B}$ and $\mathrm{C}$ contain details about some analytical computations discussed in the text. 


\section{The model}

We consider a model in which the ions are described as point charges, internal degrees of freedom being neglected. The ions interact both through a phenomenological effective potential, that accounts for the well-known shortrange repulsive quantum effects associated to the "impenetrability" of matter 1 , and through the forces due to the electromagnetic field created by all the other ions. In addition, each ion is subject to the radiation reaction force. The latter force is included because, although having a negligible magnitude, it plays a qualitatively relevant role in making the theory consistent, i.e., in ensuring the stability of the lattice. So the Newton equation of each ion (with mass $m$ and position vector $\mathbf{x}$ ) has the form

$$
m \ddot{\mathbf{x}}=\mathbf{F}^{\mathrm{rep}}+\mathbf{F}^{\mathrm{em}}+\mathbf{F}^{\mathrm{rr}},
$$

where $\mathbf{F}^{\mathrm{rep}}, \mathbf{F}^{\mathrm{em}}$ and $\mathbf{F}^{\mathrm{rr}}$ denote, respectively, the short-range repulsive force describing the interaction with neighboring ions, the electromagnetic force due to all the other ions, and the radiation reaction force.

Actually the model is studied in its linearized version. So, first of all we assume there exists an equilibrium configuration in which the ions sit on the lattice sites $\mathbf{r}_{\mathbf{h}}^{(j)}$ of the crystal under consideration, determined by the repulsive forces and the Coulomb ones. Here, as usual, $\mathbf{h} \in \mathbb{Z}^{3}$ denotes the cell, while $j=1, \ldots, n$ denotes the ion's species.

Thus, instead of the actual positions $\mathbf{x}_{\mathbf{h}}^{(j)}$ of the ions, the relevant quantities are the corresponding displacements

$$
\mathbf{u}_{\mathbf{h}}^{(j)}=\mathbf{x}_{\mathbf{h}}^{(j)}-\mathbf{r}_{\mathbf{h}}^{(j)}
$$

So, the system of Newton equations for the ions' motions takes the form

$$
m^{(j)} \ddot{\mathbf{u}}_{\mathbf{h}}^{(j)}=\mathbf{F}_{\mathbf{h},(j)}^{\mathrm{rep}}+\mathbf{F}_{\mathbf{h},(j)}^{\mathrm{em}}+\mathbf{F}_{\mathbf{h},(j)}^{\mathrm{rr}}, \quad(j=1, \ldots, n),
$$

where $m^{(j)}$ is the mass of the ions of species $(j)$.

Then the equations of motion are linearized with respect to the displacements. So, to start with, the magnetic field is completely neglected, and the

\footnotetext{
${ }^{1} \mathrm{~A}$ first-principle calculation of the repulsive short-range potential might be attempted through standard quantum many-body methods. However, here this is a minor issue, since our main concern is the correct treatment of the electromagnetic interactions.
} 
Abraham-Lorentz-Dirac radiation reaction force is taken in its nonrelativistic approximation, given by (see [6])

$$
\frac{2}{3} \frac{\left(q^{(j)}\right)^{2}}{c^{3}} \dddot{\mathbf{x}}_{\mathbf{h}}^{(j)}=\frac{2}{3} \frac{\left(q^{(j)}\right)^{2}}{c^{3}} \dddot{\mathbf{u}}_{\mathbf{h}}^{(j)}
$$

where $q^{(j)}$ is the charge of the ions of species $(j)$.

As far as the molecular repulsive forces are concerned, they may be assumed to have a phenomenological simplified form corresponding to two-body central potentials $\phi_{(j, l)}(r)$ (a priori different for each pair of species). Then, the linearization procedure amounts to computing the derivatives of the total potential, evaluated at the equilibrium configuration. Due to the short range of the repulsive forces, such derivatives rapidly approach zero as the distance between particles becomes sufficiently large (roughly, for distances larger than the linear dimension of the unit cell). In fact one may assume that the interactions occur only over the neighbors of the $(\mathbf{h}, j)$ ion. Denoting by

$$
\mathbf{r}_{\mathbf{h}-\mathbf{d}_{j}(s)}^{\left(\tau_{j}(s)\right)}, \quad s=1, \ldots, n_{j}
$$

the relative positions of the $n_{j}$ neighbors of a $j$-th species ion with respect to the ion $(\mathbf{h}, j)$, it is easy to show that the linearized repulsive forces have the form

$$
\mathbf{F}_{\mathbf{h},(j)}^{\mathrm{rep}}=\sum_{s=1}^{n_{j}}\left(\alpha_{(j), s} \Pi_{(j), s}^{\|}+\beta_{(j), s} \Pi_{(j), s}^{\perp}\right) \cdot\left(\mathbf{u}_{\mathbf{h}-\mathbf{d}_{j}(s)}^{\left(\tau_{j}(s)\right)}-\mathbf{u}_{\mathbf{h}}^{(j)}\right),
$$

where $\Pi_{(j), s}^{\|}$and $\Pi_{(j), s}^{\perp}$ are the projection operators, respectively along the direction of the neighbor separation vector $\mathbf{r}_{\mathbf{d}_{j}(s)}^{\left(j, \tau_{j}(s)\right)}$, and onto the plane normal to it. The parameters

$$
\begin{aligned}
& \alpha_{(j), s}:=\phi_{\left(j, \tau_{j}(s)\right)}^{\prime \prime}\left(\left|\mathbf{r}_{\mathbf{d}_{j}(s)}^{\left(j, \tau_{j}(s)\right)}\right|\right), \\
& \beta_{(j), s}:=\frac{\phi_{\left(j, \tau_{j}(s)\right)}^{\prime}\left(\left|\mathbf{r}_{\mathbf{d}_{j}(s)}^{\left(j, \tau_{j}(s)\right)}\right|\right)}{\left|\mathbf{r}_{\mathbf{d}_{j}(s)}^{\left(j, \tau_{j}(s)\right)}\right|},
\end{aligned}
$$

for $j=1, \ldots, n, s=1, \ldots, n_{j}$, are characteristic of the concrete crystal under consideration. Obviously, if a lattice presents a non-trivial point symmetry group, some of the parameters are likely to coincide.

Finally, we come to the linearization of the electric forces acting on each ion and due to all the other ones (the magnetic forces having been neglected). 
The electromagnetic field created by the ions is taken in the dipole approximation, i.e., as the field obtained from the Maxwell equations when the charge distribution and the current density of the sources are linearized with respect to the displacements from their equilibrium positions. So the linearized source corresponding to a certain ion, labeled by a cell index $\mathbf{p}$ and a species index $(s)$, with actual motion $\mathbf{r}_{\mathbf{p}}^{(s)}+\mathbf{u}_{\mathbf{p}}^{(s)}(t)$, is the superposition of a static source $q^{(s)}$, of zero-th order in the displacement $\mathbf{u}_{\mathbf{p}}^{(s)}$, and of a firstorder dipole source, with a dipole $\mathbf{d}(t)=q \mathbf{u}_{\mathbf{p}}^{(s)}(t)$, both at the position $\mathbf{r}_{\mathbf{p}}^{(s)}$. The solution of the corresponding inhomogeneous Maxwell equations at the spacetime point $(\mathbf{x}, t)$ is given by the superposition of the respective fields: a static spherically-symmetric Coulomb field

$$
\mathbf{E}^{[0]}(\mathbf{x}, t)=q^{(s)} \nabla \frac{1}{\left|\mathbf{x}-\mathbf{r}_{\mathbf{p}}^{(s)}\right|}
$$

and a variable dipole field

$$
\mathbf{E}^{[1]}(\mathbf{x}, t)=q^{(s)} \operatorname{curl} \operatorname{curl} \frac{\mathbf{u}_{\mathbf{p}}^{(s)}\left(t-\frac{1}{c}\left|\mathbf{x}-\mathbf{r}_{\mathbf{p}}^{(s)}\right|\right)}{\left|\mathbf{x}-\mathbf{r}_{\mathbf{p}}^{(s)}\right|},
$$

which is the one involving retardation. We are using here the standard representation of the dipole field that one finds in the classical works of Ewald [7], Oseen [8] and Born [9] on the subject. For a detailed derivation see for example [10] or [11. Such electric terms will be referred to as the "Coulomb term" and the "dipole term" respectively.

We now consider the forces resulting from the action of such fields upon a certain ion, identified by the cell index $\mathbf{h}$ and the species index $(j)$, due to all the other ones, in the linear approximation (with respect to the displacements $\mathbf{u}^{\prime}$ 's). Its actual motion is similarly denoted $\mathbf{r}_{\mathbf{h}}^{(j)}+\mathbf{u}_{\mathbf{h}}^{(j)}(t)$.

The dipole field given by (5), is already linear in $\mathbf{u}_{\mathbf{p}}^{(s)}$, hence the linear approximation of the resulting electric force amounts to evaluating it at $\mathbf{x}=$ $\mathbf{r}_{\mathbf{h}}^{(j)}$. Summing over all ions of the lattice, the force due to the dipoles is thus

$$
\mathbf{F}_{\mathbf{h},(j)}^{\operatorname{dip}}=\left.q^{(j)} \sum_{(\mathbf{p}, s)}^{\prime} q^{(s)} \operatorname{curl} \operatorname{curl} \frac{\mathbf{u}_{\mathbf{p}}^{(s)}\left(t-\frac{1}{c}\left|\mathbf{x}-\mathbf{r}_{\mathbf{p}}^{(s)}\right|\right)}{\left|\mathbf{x}-\mathbf{r}_{\mathbf{p}}^{(s)}\right|}\right|_{\mathbf{x}=\mathbf{r}_{\mathbf{h}}^{(j)}}
$$

where the prime in the sums denotes that the term $(\mathbf{p}, s)=(\mathbf{h}, j)$ is excluded. For what concerns the force due to the static Coulomb field, one has to take 
the force given by (4), evaluate it at the point $\mathbf{r}_{\mathbf{h}}^{(j)}+\mathbf{u}_{\mathbf{h}}^{(j)}(t)$, and expand it up to the first order in $\mathbf{u}_{\mathbf{h}}^{(j)}(t)$. The zeroth order term is balanced by the short range repulsive contribution, because we are evaluating the field at the equilibrium configuration, while the linear term can be written in a form which resembles that of the dipole term and highlights the electrostatic potential. In fact, the first order term is given by

$$
\mathbf{u}_{\mathbf{h}}^{(j)}(t) \cdot \nabla\left(\mathbf{E}^{[0]}\left(\mathbf{r}_{\mathbf{h}}^{(j)}, t\right)\right)
$$

where $\mathbf{E}^{[0]}$ is the gradient of the electrostatic potential. Using the identity

$$
(\mathbf{u} \cdot \nabla) \nabla \Phi=-\operatorname{curl} \operatorname{curl}(\mathbf{u} \Phi(\mathbf{x}))
$$

which holds for any scalar field $\Phi$, and taking for $\Phi$ the Coulomb potential, the linearized electric force given by the Coulomb term reads

$$
-\left.q^{(j)} q^{(s)} \operatorname{curl} \operatorname{curl} \frac{\mathbf{u}_{\mathbf{h}}^{(j)}(t)}{|\mathbf{x}-\mathbf{r}|}\right|_{\mathbf{x}=\mathbf{r}_{\mathbf{h}}^{(j)}} .
$$

So, summing over all ions of the lattice one gets

$$
\mathbf{F}_{\mathbf{h},(j)}^{\text {coul }}=-\left.q^{(j)} \sum_{(\mathbf{p}, s)}^{\prime} q^{(s)} \operatorname{curl} \operatorname{curl} \frac{\mathbf{u}_{\mathbf{h}}^{(j)}(t)}{\left|\mathbf{x}-\mathbf{r}_{\mathbf{p}}^{(s)}\right|}\right|_{\mathbf{x}=\mathbf{r}_{\mathbf{h}}^{(j)}} ;
$$

where the prime in the sum denotes that the term $(\mathbf{p}, s)=(\mathbf{h}, j)$ is excluded.

In conclusion, the linearized equations of motion for the charges of our ionic lattice read

$$
m^{(j)} \ddot{\mathbf{u}}_{\mathbf{h}}^{(j)}=\mathbf{F}_{\mathbf{h},(j)}^{\mathrm{rep}}+\mathbf{F}_{\mathbf{h},(j)}^{\mathrm{coul}}+\mathbf{F}_{\mathbf{h},(j)}^{\mathrm{dip}}+\mathbf{F}_{\mathbf{h},(j)}^{\mathrm{rr}},
$$

with

$$
\begin{aligned}
& \mathbf{F}_{\mathbf{h},(j)}^{\mathrm{rep}}=\sum_{s=1}^{n_{j}}\left(\alpha_{(j) s} \Pi_{(j) s}^{\|}+\beta_{(j) s} \Pi_{(j) s}^{\perp}\right) \cdot\left(\mathbf{u}_{\mathbf{h}-\mathbf{d}_{j}(s)}^{\tau_{j}(s)}-\mathbf{u}_{\mathbf{h}}^{(j)}\right) ; \\
& \mathbf{F}_{\mathbf{h},(j)}^{\mathrm{coul}}=-\left.q^{(j)} \sum_{(\mathbf{p}, s)}^{\prime} q^{(s)} \operatorname{curl} \operatorname{curl} \frac{\mathbf{u}_{\mathbf{h}}^{(j)}(t)}{\left|\mathbf{x}-\mathbf{r}_{\mathbf{p}}^{(s)}\right|}\right|_{\mathbf{x}=\mathbf{r}_{\mathbf{h}}^{(j)}} ; \\
& \mathbf{F}_{\mathbf{h},(j)}^{\operatorname{dip}}=\left.q^{(j)} \sum_{(\mathbf{p}, s)}^{\prime} q^{(s)} \operatorname{curl} \operatorname{curl} \frac{\mathbf{u}_{\mathbf{p}}^{(s)}\left(t-\frac{1}{c}\left|\mathbf{x}-\mathbf{r}_{\mathbf{p}}^{(s)}\right|\right)}{\left|\mathbf{x}-\mathbf{r}_{\mathbf{p}}^{(s)}\right|}\right|_{\mathbf{x}=\mathbf{r}_{\mathbf{h}}^{(j)}} ; \\
& \mathbf{F}_{\mathbf{h},(j)}^{\mathrm{rr}}=\frac{2}{3} \frac{\left(q^{(j)}\right)^{2}}{c^{3}} \dddot{\mathbf{u}}_{\mathbf{h}}^{(j)},
\end{aligned}
$$


where the prime in the sums denotes that the term $(\mathbf{p}, s)=(\mathbf{h}, j)$ is excluded. Equations (8) constitute a system of infinitely many linear equations with delay, in the unknowns $\mathbf{u}_{\mathbf{h}}^{(j)}$.

\section{Frequency-dependent dynamical matrix}

The problem of discussing equations (8) and solving them represents a formidable task. As usual, our study will be restricted to the search for generalized normal modes, i.e., oscillating modes, possibly including damped and unstable ones. Furthermore, by factoring the spatial cell dependency in the form of a plane wave, the translational symmetry of the crystal is exploited to get rid of the cell index, thus obtaining a parametric dependence on a wavevector $\mathbf{k}$ varying in the Brillouin zone.

So we substitute the plane-wave ansatz

$$
\mathbf{u}_{\mathbf{h}}^{(j)}(t)=\mathbf{U}^{(j)} e^{i \mathbf{k} \cdot \mathbf{r}_{\mathbf{h}}} e^{-i \omega t}
$$

into the equations of motion and look at the corresponding equations. One obviously has

$$
\begin{aligned}
m^{(j)} \ddot{\mathbf{u}}_{\mathbf{h}}^{(j)} & =-\omega^{2} m^{(j)} \mathbf{U}^{(j)} e^{i \mathbf{k} \cdot \mathbf{r}_{\mathbf{h}}} e^{-i \omega t} ; \\
\mathbf{F}_{\mathbf{h},(j)}^{\mathrm{coul}} & =-e^{i \mathbf{k} \cdot \mathbf{r}_{\mathbf{h}}} e^{-i \omega t} q^{(j)} \sum_{(\mathbf{p}, s)}^{\prime} q^{(s)} \text { curl curl }\left.\frac{\mathbf{U}^{(j)}}{\left|\mathbf{x}-\mathbf{r}_{\mathbf{p}}^{(s)}\right|}\right|_{\mathbf{x}=\mathbf{r}_{\mathbf{h}}^{(j)}} ; \\
\mathbf{F}_{\mathbf{h},(j)}^{\mathrm{rr}} & =-i \omega^{3} \frac{2}{3} \frac{\left(q^{(j)}\right)^{2}}{c^{3}} \mathbf{U}^{(j)} e^{i \mathbf{k} \cdot \mathbf{r}_{\mathbf{h}}} e^{-i \omega t} .
\end{aligned}
$$

and furthermore, as usual in lattice dynamics,

$$
\begin{aligned}
\mathbf{F}_{\mathbf{h},(j)}^{\mathrm{rep}} & =\sum_{s=1}^{n_{j}}\left(\alpha_{(j) s} \Pi_{(j) s}^{\|}+\beta_{(j) s} \Pi_{(j) s}^{\perp}\right) \cdot\left(\mathbf{U}^{\tau_{j}(s)} e^{i \mathbf{k} \cdot\left(\mathbf{r}_{\mathbf{h}}-\mathbf{r}_{\mathbf{d}_{j}(s)}\right)} e^{-i \omega t}-\mathbf{U}^{(j)} e^{i \mathbf{k} \cdot \mathbf{r}_{\mathbf{h}}} e^{-i \omega t}\right) \\
& =e^{i \mathbf{k} \cdot \mathbf{r}_{\mathbf{h}}} e^{-i \omega t} \sum_{s=1}^{n_{j}}\left(\alpha_{(j) s} \Pi_{(j) s}^{\|}+\beta_{(j) s} \Pi_{(j) s}^{\perp}\right) \cdot\left(\mathbf{U}^{\left(\tau_{j}(s)\right)} e^{-i \mathbf{k} \cdot \mathbf{r}_{\mathbf{d}_{j}(s)}}-\mathbf{U}^{(j)}\right)
\end{aligned}
$$

For what concerns the dipole terms, instead, one obtains a qualitatively different contribution, because, due to retardation, there appear terms which 
depends on $\omega$ not simply through the factor $\exp (-i \omega t)$. In fact one has

$$
\begin{aligned}
\mathbf{F}_{\mathbf{h},(j)}^{\operatorname{dip}} & =\left.q^{(j)} \sum_{(\mathbf{p}, s)}^{\prime} q^{(s)} \operatorname{curl} \operatorname{curl} \frac{\mathbf{U}^{(s)} e^{i \mathbf{k} \cdot \mathbf{r}_{\mathbf{p}}} e^{-i \omega t-\frac{\omega}{c}\left|\mathbf{x}-\mathbf{r}_{\mathbf{p}}^{(s)}\right|}}{\left|\mathbf{x}-\mathbf{r}_{\mathbf{p}}^{(s)}\right|}\right|_{\mathbf{x}=\mathbf{r}_{\mathbf{h}}^{(j)}} \\
& =\left.e^{i \mathbf{k} \cdot \mathbf{r}_{\mathbf{h}}} e^{-i \omega t} q^{(j)} \sum_{(\mathbf{p}, s)}^{\prime} q^{(s)} \operatorname{curl} \operatorname{curl} \frac{\mathbf{U}^{(s)} e^{i \mathbf{k} \cdot\left(\mathbf{r}_{\mathbf{p}}-\mathbf{r}_{\mathbf{h}}\right)} e^{-\frac{\omega}{c}\left|\mathbf{x}-\mathbf{r}_{\mathbf{p}}^{(s)}\right|}}{\left|\mathbf{x}-\mathbf{r}_{\mathbf{p}}^{(s)}\right|}\right|_{\mathbf{x}=\mathbf{r}_{\mathbf{h}}^{(j)}} \\
& =\left.e^{i \mathbf{k} \cdot \mathbf{r}_{\mathbf{h}}} e^{-i \omega t} q^{(j)} \sum_{(\mathbf{p}, s)}^{\prime} q^{(s)} \operatorname{curl} \operatorname{curl} \frac{\mathbf{U}^{(s)} e^{-i \mathbf{k} \cdot \mathbf{r}_{\mathbf{p}}} e^{-\frac{\omega}{c}|\mathbf{x}|}}{|\mathbf{x}|}\right|_{\mathbf{x}=\mathbf{r}_{\mathbf{p}}^{(j, s)}},
\end{aligned}
$$

where in the last line we simply replaced the dummy index $\mathbf{h}-\mathbf{p}$ by $\mathbf{p}$. So, substituing the above relations into equation (8), after dividing by the common factor $e^{i \mathbf{k} \cdot \mathbf{r}_{\mathbf{h}}} e^{-i \omega t}$ one gets to the set of linear equations in the unknown $\mathbf{U}^{(j)}$

$$
\begin{aligned}
-m^{(j)} \omega^{2} \mathbf{U}^{(j)} & =\sum_{s=1}^{n_{j}}\left(\alpha_{(j), s} \Pi_{(j), s}^{\|}+\beta_{(j), s} \Pi_{(j), s}^{\perp}\right) \cdot\left(\mathbf{U}^{\left(\tau_{j}(s)\right)} e^{-i \mathbf{k} \cdot \mathbf{r}_{\mathbf{d}_{j}(s)}}-\mathbf{U}^{(j)}\right)+ \\
& -\left.q^{(j)} \sum_{(\mathbf{p}, s)}^{\prime} q^{(s)} \operatorname{curl} \operatorname{curl} \frac{\mathbf{U}^{(j)}}{|\mathbf{x}|}\right|_{\mathbf{x}=\mathbf{r}_{\mathbf{p}}^{(j, s)}}+ \\
& +\left.q^{(j)} \sum_{(\mathbf{p}, s)}^{\prime} q^{(s)} \operatorname{curl} \operatorname{curl} \frac{\mathbf{U}^{(s)} e^{-i \mathbf{k} \cdot \mathbf{r}_{\mathbf{p}}} e^{i \frac{\omega}{c}|\mathbf{x}|}}{|\mathbf{x}|}\right|_{\mathbf{x}=\mathbf{r}_{\mathbf{p}}^{(j, s)}}++i \frac{2}{3} \frac{\left(q^{(j)}\right)^{2}}{c^{3}} \omega^{3} \mathbf{U}^{(j)} .
\end{aligned}
$$

With $j=1,2, \ldots, n$, we have in all $3 n$ linear equations, in which $\mathbf{k}$ enters as a parameter and $\omega$ as an unknown. Such equations can be written symbolically in the form

$$
\begin{aligned}
&-m^{(j)} \omega^{2} \mathbf{U}^{(j)}= \\
& \sum_{s=1}^{n}\left[\hat{\mathcal{P}}_{j s}(\mathbf{k})+\hat{\mathcal{C}}_{j s}+\hat{\mathcal{D}}_{j s}(\mathbf{k}, \omega)\right] \cdot \mathbf{U}^{(s)}+i \frac{2}{3} \frac{\left(q^{(j)}\right)^{2}}{c^{3}} \omega^{3} \mathbf{U}^{(j)},
\end{aligned}
$$

having denoted by $\mathcal{P}=\left\{\hat{\mathcal{P}}_{j s}\right\}, \mathcal{C}=\left\{\hat{\mathcal{C}}_{j s}\right\}, \mathcal{D}=\left\{\hat{\mathcal{D}}_{j s}\right\}$ respectively the matrix of the short-range repulsive forces, that of the Coulomb forces, and that of the dipole ones. In short, we can also write the equations for the normal 
modes in the form

$$
-m^{(j)} \omega^{2} \mathbf{U}^{(j)}=\sum_{s=1}^{n} \hat{\mathcal{A}}_{j s}(\mathbf{k}, \omega) \cdot \mathbf{U}^{(s)}
$$

which involves a dynamical matrix $\mathcal{A}$ in a way apparently similar to that occurring in the case of the "instantaneous" Coulomb model. A sharp difference is however that the dynamical matrix $\mathcal{A}$ now depends on the unknown $\omega$, besides on the wavevector parameter $\mathbf{k}$. Such a dependency is obviously contained in the dipole part. This is a peculiar and remarkable consequence of retardation, which entails that equation (13) does not give rise to a standard secular equation. Denoting by $\mathcal{M}=\operatorname{diag}\left(m_{1}, \ldots, m_{n}\right)$ the mass matrix, the analogue of the secular equation presently takes the form

$$
\operatorname{det}\left(\omega^{2} \mathcal{M}+\mathcal{A}(\mathbf{k}, \omega)\right)=0 \text {. }
$$

This makes the dispersion relations more difficult to compute. Due to the $\omega$-dependency of the dynamical matrix, the solutions cannot be worked out through standard diagonalization methods of linear algebra, and a more general numerical algorithm is required.
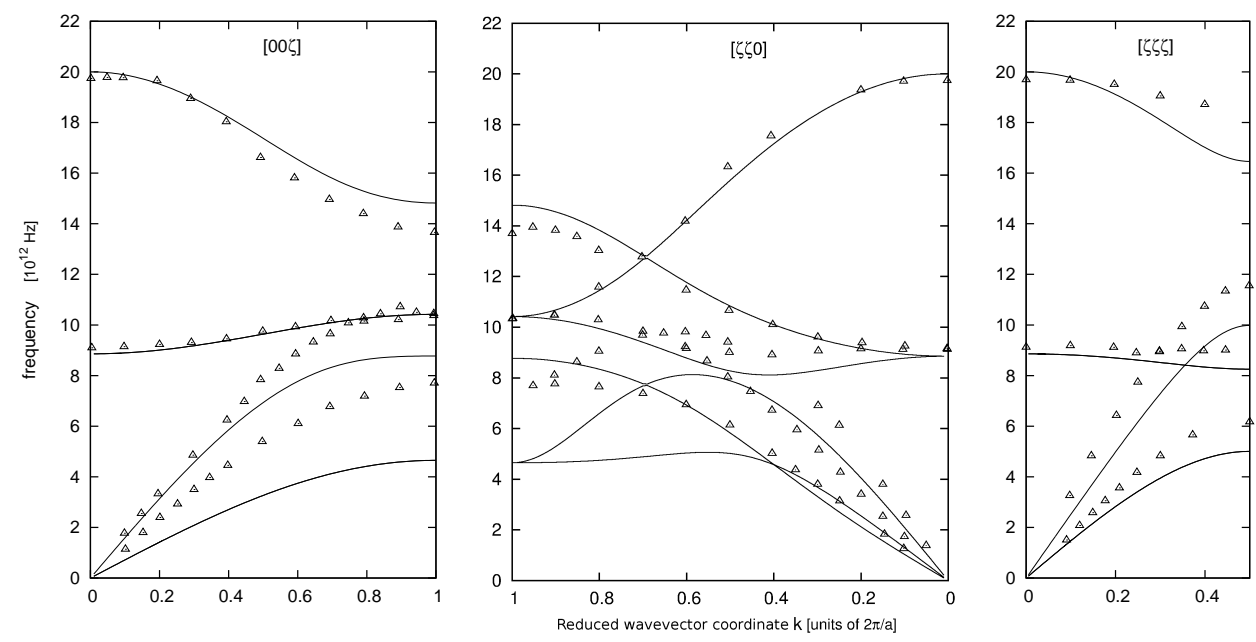

Figure 1: Dispersion relations along three directions of high symmetry in LiF. Solid curves are the theoretical predictions, and triangles the experimental data taken from [14] 
Moreover, we see that the $\omega$-dependency of the entries of $\mathcal{A}$ is by no means simple; actually it is not algebraic, neither can it be expressed in terms of elementary functions. Thus, it is not even possible to foresee how many solutions, for each value of $\mathbf{k}$ do exist. The number of branches and their topology may vary in a substantial way with respect to the "mechanical or instantaneous" case, which displays $3 n-3$ optical branches and 3 acoustic ones. This is indeed what makes the existence of polaritonic branches possible.

One sees that the instantaneous Coulomb limit case is formally obtained by taking the limit $\omega \rightarrow 0$ (or equivalently $c \rightarrow \infty$ ) in the dipole field in (11). In such a limit, the sum of the Coulomb term and of the dipole one gives simply an additional "mechanical" term, completely analogous to the molecular repulsive one, the only difference being that the long range of the Coulomb forces makes a resummation procedure necessary. However, it turns out that this limit misses most of the physics involved in the region about the e.m. dispersion curve $\omega=c k$, which is the primary goal of the present discussion.

The sums over the lattice, which define the electric fields in (11), are obviously ill-defined, due to the long range of the e.m. interactions. A precise meaning is assigned to them through the well-known Ewald's summation procedure [7]. This transforms each conditionally convergent series into the sum of two rapidly convergent ones, which account for the "short-distance" part and the "long-distance" part of the interactions respectively. The use of such a method is crucial, for it provides a deep insight into the physical aspect of the problem. It turns out that only the term describing longdistance interactions is substantially modified by the $\omega$-dependency (i.e., by retardation), and that such dependency is important only in the region about the e.m. dispersion curve $\omega=c k$.

The details of the Ewald's summation method can be found either in his original paper [7] or in more recent works (e.g., [12]). A compact exposition can also be found in the appendix of [13]. A slight generalization of the method had to be devised in order to deal with the dipole fields. This is discussed in Appendix B. It turns out that, once the Ewald resummation has been performed, the dipole part of the dynamical matrix takes, for $j \neq l$, the 
structure

$$
\begin{aligned}
\hat{\mathcal{D}}_{j l}=q^{(j)} q^{(l)}\{ & \left.\frac{4 \pi}{\left|V_{c}\right|} \sum_{\mathbf{m} \in \mathbb{Z}^{3}} \frac{e^{-\frac{1}{4 \delta^{\prime 2}}\left(\left|\mathbf{q}_{\mathbf{m}}-\mathbf{k}\right|^{2}-\frac{\omega^{2}}{c^{2}}\right)}}{\left|\mathbf{q}_{\mathbf{m}}-\mathbf{k}\right|^{2}-\frac{\omega^{2}}{c^{2}}} \hat{\mathcal{R}}\left[e^{+i\left(\mathbf{q}_{\mathbf{m}}-\mathbf{k}\right) \cdot \mathbf{x}}\right]\right|_{\mathbf{x}=\mathbf{b}^{(j, l)}} \\
& \left.+\left.\sum_{\mathbf{h} \in \mathbb{Z}^{3}} e^{-i \mathbf{k} \cdot \mathbf{r}_{\mathbf{h}}} \frac{2}{\sqrt{\pi}} \int_{\delta^{\prime}}^{+\infty} d \eta e^{\frac{\omega^{2}}{4 c^{2}} \frac{1}{\eta^{2}}} \hat{\mathcal{R}}\left[e^{-|\mathbf{x}|^{2} \eta^{2}}\right]\right|_{\mathbf{x}=\mathbf{r}_{\mathbf{h}}^{(j, l)}}\right\},
\end{aligned}
$$

where the first term describes the field associated to the far charges, while the second one accounts for the short-range part of the interactions. The notations are as follows: $\mathbf{r}_{\mathbf{h}}$ runs over the direct lattice, $\mathbf{q}_{\mathbf{m}}$ runs over the reciprocal lattice; $\mathbf{b}^{(j)}$ for $j=1, \ldots, n$ are the positions of the ions within a unit cell $\left(\mathbf{r}_{\mathbf{h}}^{(j)}=\mathbf{r}_{\mathbf{h}}+\mathbf{b}^{(j)}\right) ; V_{c}$ is the region of a cell and $\left|V_{c}\right|$ its volume. Furthermore, $\hat{\mathcal{R}}$ is the matrix form of the differential operator curl curl: for a scalar field $f$ one has

$$
\hat{\mathcal{R}}[f(\mathbf{x})]=\hat{H}[f(\mathbf{x})]-\Delta[f(\mathbf{x})] \hat{\mathbb{I}}_{3}
$$

where $\hat{H}[f]$ is the Hessian of $f$ (the matrix of its second derivatives), $\Delta$ the Laplacian and $\hat{\mathbb{I}}_{3}$ the identity matrix.

For $j=l$ the matrix element has just the same form, apart from the fact that the unphysical self-interaction term must be subtracted. Here a very interesting fact occurs: such a subtracted term gives rise to a non-hermitian part for the dipole term matrix, that is exactly canceled by the radiation reaction term (the last one in (11), which is clearly non-hermitian, too). Indeed, as shown in Appendix $\mathrm{A}$, for all $j, s=1, \ldots, n$ one has

$$
\frac{1}{2}\left(\hat{\mathcal{D}}_{j s}(\mathbf{k}, \omega)-\hat{\mathcal{D}}_{s j}^{\dagger}(\mathbf{k}, \omega)\right)+i \frac{2}{3} \frac{q^{(j)}}{c^{3}} \omega^{3} \delta_{j s} \hat{\mathbb{I}}_{3}=0
$$

Hence, the complete dynamical matrix $\mathcal{A}$ is hermitian, so that only real frequencies are allowed. We thus see that the radiation reaction force is vital to the stability of the lattice: a nonvanishing imaginary part in the frequency would correspond to damped or unstable oscillations. This a priori unexpected cancellation, first realized by Oseen [8], and then rediscovered in [15, 16], might seem "accidental" at first sight. The proof of such a cancellation, given in appendix A, shows its deep meaning, first pointed out by Wheeler and Feynman[5]. 
The splitting parameter $\delta^{\prime}$ in (15), involved in Ewald's method, is arbitrary, to be chosen so that both series converge rapidly. If it is taken of the order of the inverse first-neighbor interatomic distance, one sees that

$$
\frac{\omega}{2 c \eta} \lesssim 10^{-5} \quad \text { for all } \eta>\delta^{\prime},
$$

if one takes $\omega$ in the range of the typical frequencies of crystal dynamics, i.e., $\omega \simeq 10^{13} \mathrm{~Hz}$. Hence we may safely take $\omega=0$ in the corresponding exponential in the second term: the $\omega$-dependency of the short-distance part of the interactions is negligible, so that they can be considered as instantaneous. Instead, the first term, which describes the long-distance part of the interactions, is strongly frequency-dependent: the term $\mathbf{m}=\mathbf{0}$ has in fact a pole at $\omega=c k$, i.e., along the e.m. dispersion line.

This is the main mathematical effect of retardation: the part of the dynamical matrix which describes the interaction with the far charges strongly depends on the frequency $\omega$ near the e.m. dispersion curve $\omega=c k$. Hence, very different predictions are expected with respect to the instantaneous Coulomb model. In the following, we show that such a feature leads indeed to retrieve the polariton curves.

\section{Polaritons in a rock salt lattice}

At this point we are no longer able to proceed any further on a general discussion. In order to check concretely the predictions of the model, a numerical study is needed, and so we concentrate on a specific crystal structure. In view of a comparison with experimental data, we choose the rock salt structure, which is in fact very simple and is shared by the most common alkali halides. Concretely, the comparison with the experimental data was performed for the case of Lithium Floride, LiF. So, we first have to explicitly write down the dynamical matrix $\mathcal{A}$ in general, which is done in appendix [C, Then, the expressions thus found have to be specialized to the case of the rock salt lattice.

In order to solve numerically the generalized secular equation (14), with the dynamical matrix $\mathcal{A}$ now computed for a generic rock salt lattice, we devised a very general and straightforward algorithm. The overall idea is the following. The wavevector $\mathbf{k}$ is let run along certain directions from the zone center to the zone boundary, and the frequency $\omega$ is let vary in a suitable 
range. Then, considering both $\mathbf{k}$ and $\omega$ as parameters, the dynamical matrix is numerically evaluated, and a generalized diagonalization is performed, by determining the six values $\lambda_{i}(\mathbf{k}, \omega)$ such that $\operatorname{det}\left(\lambda_{i} \mathcal{M}+\mathcal{A}(\mathbf{k}, \omega)\right)=0$. Eventually, leaving $\mathbf{k}$ only as a parameter, the roots of the equations

$$
\lambda_{i}(\mathbf{k}, \omega)=-\omega^{2}
$$

are numerically determined for each $i=1, \ldots, 6$. This yields the admissible excitation frequencies of the lattice for the given wavevector $\mathbf{k}$. In particular, as previously mentioned, the pole in the matrix elements along the line $\omega=c k$ gives rise to a corresponding pole for certain eigenvalues (as functions of $\omega$ ), and hence the number of solutions of (16) doubles: at variance with the instantaneous Coulomb model, we have more than $3 n$ branches. For values of $\mathbf{k}$ and $\omega$ far from the line $\omega=c k$ the whole procedure is actually redundant, because the instantaneous approximation is perfectly suitable.

Now, the dispersion curves thus found still depend on the three free parameters entering the model, which are determined by a best fit with the experimental phonon curves. The best fit thus obtained is exhibited in figure 1. In table 4 the numerical values employed for the parameters entering the model are collected.

The three panels give $\omega$ versus $k$ for the three high-symmetry directions of $\mathbf{k}(0,0,1)$ (left), $(1,1,0)$ (center) and $(1,1,1)$ (right) for the whole Brillouin zone. The triangles are the experimental values taken from [14], while the continuous lines give the theoretical curves. The global agreement over the whole Brillouin zone seems to us to be fairly good, in consideration of the simplicity of the model, and of the small number (three) of free parameters. In fact, one can notice that the fit is not so good for the acoustic branches, especially for high $k$. A better fit over the whole Brillouin zone was actually obtained in the paper [14], using second-nearest-neighbor short range forces, in a model involving seven free parameters. So, presumably, an analogous better fit could have been obtained by us too by making recourse to a more refined model for the short range forces. However, we did not care for this, because our main goal is to exhibit the occurrence of polaritons in the simplest possible way.

Now, the polaritonic branches cannot be seen in figure 1 because they are squeezed along the ordinate axis. They are exhibited in figure 2, in which a zoom of the left panel of figure 1 is performed, by enlarging by a factor $10^{5}$ the axis of the abscissæ. 


\begin{tabular}{cc}
\hline Parameter & Value \\
\hline$\alpha$ & -0.19 \\
$\beta$ & +0.028 \\
$q$ & 0.6 \\
$a$ & 4.02 \\
$m^{+}$ & 0.005 \\
$m^{-}$ & 0.0136 \\
\hline
\end{tabular}

Table 1: Parameters for LiF (units: $\AA, 10^{-13} s, e$ ). The first three ones (the force constants $\alpha, \beta$ and the effective charge of both ions $q$ ) have been obtained by fitting the experimental phonon dispersion curves taken from [14. For the lattice parameter $a$ and the masses $m^{+}, m^{-}$the commonly accepted values have been employed.

As expected, near the line $\omega=c k$, the number and the topology of the branches are drastically different with respect to those of the instantaneous approximation, and presents a pattern displaying polaritonic curves. As the acoustic branches are now squeezed on the abscissæ axis, one should look at the optical branches, which in figure 1 intersect the axis of the ordinates at $\nu \approx 910^{12} \mathrm{~Hz}$ (the two transverse ones) and at $\nu \approx 2010^{12} \mathrm{~Hz}$ (the longitudinal one). One sees that the two transverse branches actually go to zero, with a slope around $c / 2.27$, whereas there appear two new branches, actually degenerate, which start from the fundamental frequency of the longitudinalmodes branch at $k=0$ and are asymptotic to the e.m. dispersion line $\omega=c k$. Namely, the transverse optical branch (which actually represents the two transverse-mode curves) splits into a lower branch, which approaches zero, and an upper one. Such a phenomenon is a peculiar effect of retardation, which strongly couples the radiation field of far charges to the vibrational modes.

The longitudinal-modes branch, instead, is untouched by retardation. This is due to the fact that the electromagnetic waves, being transverse, do not couple with longitudinal modes. Indeed, the only terms in (15) which are affected by retardation, i.e., the terms $\mathbf{m}=\mathbf{0}$ of the sums over the reciprocal lattice, contain a projection onto the plane orthogonal to the wavevector $\mathbf{k}$, which yields zero when applied to longitudinal displacements $\mathbf{U}^{(l)}$.

In figure 2 are reported also (triangles) the experimental values taken from the work [17], and one sees that the agreement is pretty good for small 


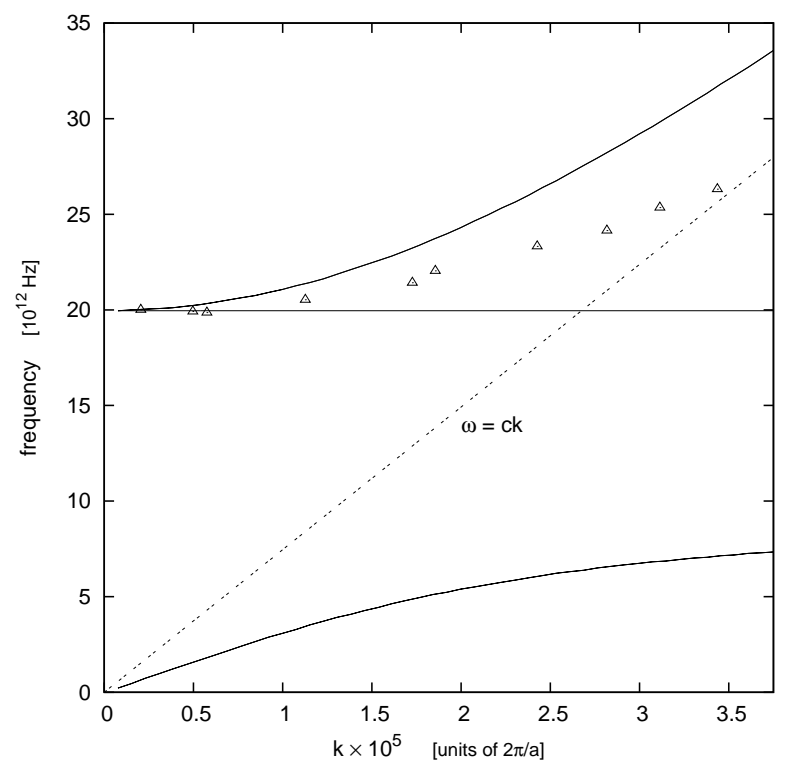

Figure 2: Zoom of the central panel of figure 1 for small $k$, exhibiting polaritonic branches. Solid curves are the theoretical predictions, dashed line is the curve $\omega=c k$, while triangles are the experimental data at $420 \mathrm{~K}$, taken from [17]. Acoustic branches superimpose to the axis $\omega=0$

$k$, while it is not so good for the asymptotic behavior at larger $k$. Actually we found that the agreement in the small $k$ region depends very critically on the value of the effective charge, which had to be carefully chosen. Instead, the partial discrepancy in the high $k$ region appears to be rather due to a deficiency of the model itself, inasmuch as the ions are dealt with as point charges without any internal structure. In order to appreciate this fact one should notice that, as better discussed in the following section, the dispersion curves for the lattice vibrations coincide with the dispersion curves of the e.m. field propagating inside the crystal. In other terms, the slope of the lower branch in the low-frequency region of figure 2 should coincide with the speed of e.m. field propagation inside the crystal, i.e., with

$$
c / \sqrt{\varepsilon}
$$

where $\varepsilon$ is the static dielectric constant of the medium. In the usual macroscopic treatments of electromagnetism and optics, such a constant is a phenomenological parameter related to the polarizability of the system. Here, 
instead, it arises in a natural way as a consequence of the microscopic dynamics, and its value is deduced from the geometrical structure of the lattice and from the physical parameters which characterize the ionic crystal under consideration (masses, charges and the repulsive force constants). In our case we found $\varepsilon \approx 5.3$, while experimentally one finds $\varepsilon \approx 9$. We expect that this discrepancy should be attributed to the approximation of considering the ions as point particles, thus neglecting polarizability. Now, we expect that this approximation should be responsible also for the discrepancy concerning the upper transverse polariton high-frequency slope, which here is $c$, while being experimentally smaller. Indeed the modification of the speed of the e.m. field at the optical frequencies should be due to the interactions with the electrons, which is neglected in the present model.

We also point out that between the upper and the lower transverse branches there occurs a frequency gap, which should correspond to the frequency of the infrared residual rays.

\section{Deduction of macroscopic optics}

So far we have deduced, calculated and discussed the mere vibrational properties of ionic lattices, by determining their normal modes when the electromagnetic forces are taken into account. In this section we show how such microscopic lattice dynamics gives rise to a macroscopic propagation of electromagnetic fields across the lattice, in agreement with the laws of macroscopic optics.

The field $\mathbf{E}(\mathbf{x}, t)$ propagating across the lattice is obtained in the linear approximation by evaluating the superposition of the variable electric fields generated by the oscillations of the dipoles at each lattice point, which gives

$$
\mathbf{E}(\mathbf{x}, t)=\sum_{(\mathbf{h}, j)} \operatorname{curl} \operatorname{curl}\left(q^{(j)} \frac{\mathbf{u}_{\mathbf{h}}^{(j)}\left(t-\frac{1}{c}\left|\mathbf{x}-\mathbf{r}_{\mathbf{h}}^{(j)}\right|\right)}{\left|\mathbf{x}-\mathbf{r}_{\mathbf{h}}^{(j)}\right|}\right) .
$$

Let us now suppose that the ions are oscillating according to a normal mode, identified by $\omega$ and $\mathbf{k}$ : the law of motion of each dipole is thus

$$
\mathbf{u}_{\mathbf{h}}^{(j)}(t)=\mathbf{U}^{(j)} e^{i \mathbf{k} \cdot \mathbf{r}_{\mathbf{h}}} e^{-i \omega t},
$$


for some definite amplitude vectors $\left\{\mathbf{U}^{(j)}(\mathbf{k}, \omega)\right\}_{j=1, \ldots, n}$. The field is then

$$
\mathbf{E}(\mathbf{x}, t)=
$$

or equivalently

$$
\mathbf{E}(\mathbf{x}, t)=e^{-i \omega t} \operatorname{curl} \operatorname{curl}\left[\sum_{j=1}^{n} q^{(j)} \Psi\left(\mathbf{x}-\mathbf{b}^{(j)}\right) \mathbf{U}^{(j)}\right]
$$

where we have introduced

$$
\Psi(\mathbf{x}):=\sum_{\mathbf{p} \in \mathbb{Z}^{3}} e^{+i \mathbf{k} \cdot \mathbf{r}_{\mathbf{p}}} \frac{e^{i \frac{\omega}{c}\left|\mathbf{x}-\mathbf{r}_{\mathbf{p}}\right|}}{\left|\mathbf{x}-\mathbf{r}_{\mathbf{p}}\right|}
$$

As shown in Appendix $B$, we can now carry on the manipulation on $\Psi(\mathbf{x})$, leading to a sum of rapidly convergent series, namely

$$
\begin{aligned}
\Psi(\mathbf{x}) & =\Psi_{1}(\mathbf{x})+\Psi_{2}(\mathbf{x}):=\frac{4 \pi}{\left|V_{c}\right|} \sum_{\mathbf{m} \in \mathbb{Z}^{3}} \frac{e^{-\frac{1}{4 \delta^{\prime 2}}\left(\left|\mathbf{q}_{\mathbf{m}}-\mathbf{k}\right|^{2}-\frac{\omega^{2}}{c^{2}}\right)}}{\left|\mathbf{q}_{\mathbf{m}}-\mathbf{k}\right|^{2}-\frac{\omega^{2}}{c^{2}}} e^{i\left(\mathbf{k}-\mathbf{q}_{\mathbf{m}}\right) \cdot \mathbf{x}}+ \\
& +\sum_{\mathbf{h} \in \mathbb{Z}^{3}} e^{i \mathbf{k} \cdot \mathbf{r}_{\mathbf{h}}} \frac{2}{\sqrt{\pi}} \int_{\delta^{\prime}}^{+\infty} e^{\frac{\omega^{2}}{4 c^{2}} \frac{1}{\eta^{2}}} e^{-\left|\mathbf{x}-\mathbf{r}_{\mathbf{h}}\right|^{2} \eta^{2}} d \eta
\end{aligned}
$$

Exploiting the algebraic identity

$$
a^{2}-b^{2}=(a \pm i b)^{2} \mp 2 i a b
$$

in the exponent of the integrand, it is convenient to rewrite the second part $\Psi_{2}(\mathbf{x})$ of $\Psi$ in the remarkable form

$$
\Psi_{2}(\mathbf{x}) e^{-i \omega t}=\sum_{\mathbf{h} \in \mathbb{Z}^{3}}\left(e^{i \mathbf{k} \cdot \mathbf{r}_{\mathbf{h}}} \frac{2}{\sqrt{\pi}} \int_{\delta^{\prime}}^{+\infty} e^{\left(\frac{\omega}{2 c} \frac{1}{\eta}-i\left|\mathbf{x}-\mathbf{r}_{\mathbf{h}}\right| \eta\right)^{2}} d \eta\right) e^{i\left(\frac{\omega}{c}\left|\mathbf{x}-\mathbf{r}_{\mathbf{h}}\right|-\omega t\right)} .
$$

This expression highlights that, for a not too small splitting parameter $\delta^{\prime}$ (say, comparable to the inverse interatomic first-neighbor distance), $\Psi_{2}$ is roughly the superposition of spherical waves coming from the neighboring 
sites and propagating at speed $c$.

The function $\Psi_{1}(\mathbf{x})$ can instead be written as

$$
\Psi_{1}(\mathbf{x}) e^{-i \omega t}=\left(\frac{4 \pi}{\left|V_{c}\right|} \sum_{\mathbf{m} \in \mathbb{Z}^{3}} \frac{e^{-\frac{1}{4 \delta^{\prime 2}}\left(\left|\mathbf{q}_{\mathbf{m}}-\mathbf{k}\right|^{2}-\frac{\omega^{2}}{c^{2}}\right)}}{\left|\mathbf{q}_{\mathbf{m}}-\mathbf{k}\right|^{2}-\frac{\omega^{2}}{c^{2}}} e^{-i \mathbf{q}_{\mathbf{m}} \cdot \mathbf{x}}\right) e^{i(\mathbf{k} \cdot \mathbf{x}-\omega t)}
$$

The term in brackets is a smooth quasiperiodic function over the direct lattice. Thus $\Psi_{1}$ looks like a plane wave characterized by the phonon wavevector $\mathbf{k}$ and the frequency $\omega$, hence propagating at the speed

$$
v=\frac{\omega}{|\mathbf{k}|}=: \frac{c}{n_{b}(\mathbf{k})} .
$$

The refraction index $n$ depends on the considered normal mode, identified by a wavevector $\mathbf{k}$ plus a branch label $b=1,2, \ldots$, corresponding to the specific branch considered.

Substituting such expressions of $\Psi_{1}$ and $\Psi_{2}$ into (17) and computing the effect of curl curl on them, we find the explicit form of the propagating field. We see that the term originating from (19) varies over a microscopic scale (i.e., one comparable to the linear dimensions of a primitive cell), whereas the term resulting from (20) is much "smoother". If we consider that a reasonable measuring instrument should be necessarily macroscopic, hence much larger than the atomic scale, then only the latter field should be observable. Therefore, we name the former microscopic field, and the latter macroscopic field. Such a distinction is significant at macroscopic scales, i.e., in the usual frame of the elementary (phenomenological) treatments of optics.

This result means that at least a relevant part of the infrared dispersion phenomenology in ionic crystals can be deduced from the vibrational dispersion relations: indeed through equation (21) the refraction index can be expressed as a function of frequency. The interaction between radiation and matter (or phonon-photon coupling, in the quantum picture) can be interpreted in terms of such relations.

\section{Final considerations}

So, by studying a microscopic classical model of an ionic crystal with the ions dealt with as point charges, we have shown that the retarded action of the far ions is responsible for the splitting of the dispersion curves about 
the e.m. dispersion line $\omega=c k$, i.e., for the existence of polaritons in ionic crystals.

A fairly good quantitative agreement between the theoretical polaritonic curves and the experimental data for Lithium Fluoride is obtained directly from microscopic dynamics, without further ad hoc assumptions or the use of any fitting parameters, apart from the three parameters (effective charge of the ions and two constants entering the repulsive forces) related to the non-retarded part of the problem. In any case, polaritons come out automatically in virtue of retardation, without the need of introducing any new parameter, once the instantaneous part of the problem has been settled. For what concerns the choice of the free parameters, one may notice that they could also be determined from experimental data not related to the dispersion curves, as for example thermodynamic quantities such as the internal energy or data on the infrared absorption (see [18]).

One may now ask whether an explanation of dispersion can in some analogous way be given also for other types of crystal insulators. The simplest model is obtained by considering a lattice of pure dipoles. Such a model was already studied in the work [16], and a phenomenon analogous to that of existence of polaritonic curves was observed.

We finally add now a comment concerning the treatment of the problem given in the book of Born and Huang[1. We already mentioned that such authors predicted the existence of polaritons in the first part of the book, where the problem is discussed in terms of macroscopic polarization fields. It seems however that a proof is lacking in the second part of the book, which is devoted to a microscopic discussion of the problem. Apparently this is due to the fact that, in discussing the secular equation, the authors do not introduce explicitly $\omega$ as an unknown of the problem, limiting themselves to introduce the ansatz $\omega=(c / n) k$. This entails that the upper polaritonic branch cannot be detected. Moreover, at page 334, they explicitly say that "The last term (i.e., the retarded one) can be ignored".

Acknowledgement: We thank Giuseppe Pastori Parravicini. Having read the papers [15] and [16], where retardation was taken into account in microscopic models involving internal dipoles only, he suggested that polaritons may be proven to exist by analogous methods, if one considers a model involving the displacements of the ions. 


\section{A The Wheeler-Feynman identity and the sta- bility of the lattice}

In discussing the secular equation, it was already pointed out that the term (2), due to the radiation reaction force entering the Newton equation for each ion, exactly cancels the non-hermitian part of the dynamical matrix, so that one is left with a hermitian dynamical matrix. This has the consequence that only real frequencies $\omega$ (i.e. stable non-damped oscillations) are allowed, so that the lattice can exist. As mentioned in [15], such a cancellation was first pointed out by Oseen 8 in the year 1916. Note that a priori such a cancellation is by no means evident nor obvious: when radiation effects are taken into account, one might expect that non-trivial energy exchanges occur, and damped oscillations may arise due to uncompensated energy losses. It should be noted that, if $\omega$ has a positive imaginary part, the dipole term series describing the retarded interaction diverges. The actual role of the radiation reaction force deserves thus to be pointed out: its inclusion in the equations of motion is vital in order to make the lattice stable, and in allowing for the existence itself of a dispersion relation; omitting this term would lead to a substantial inconsistency of the model.

It might seem that the Oseen cancellation occurring for the non-hermitian part of the dynamical matrix, arises "accidentally". Here we provide a proof, more significant than the straightforward computation implicitly carried out in the text. The present proof shows that the origin of the cancellation is actually deeper, and can ultimately be ascribed to the symmetry of electrodynamics with respect to time inversion.

In fact, we prove here that the present model of ionic crystal satisfies the following identity, first proposed by Wheeler and Feynman[5]:

$$
\sum_{k}\left(F_{\text {ret }}^{k}(\mathbf{x}, t)-F_{\text {adv }}^{k}(\mathbf{x}, t)\right)=0
$$

where the summation index $k$ runs over all the charged particles of the system and $F$ is the e.m. field tensor 2 This evidently points out the symmetrical role played by the retarded and the advanced solutions of the Maxwell equations. We will then show that this identity actually implies the Oseen cancellation.

\footnotetext{
${ }^{2}$ Alternatively, we might say that the crystal has the property of being a WheelerFeynman complete absorber.
} 
First of all, it is clear that the zeroth-order Coulomb fields trivially satisfy the above identity, because they are independent of time. In addition, recall that we are neglecting magnetic fields, which give second-order effects. Thus, verifying (22) amounts to showing that

$$
\sum_{(\mathbf{p}, s)}\left(\mathbf{E}_{\mathbf{p}, s}^{[1], \text { ret }}(\mathbf{x}, t)-\mathbf{E}_{\mathbf{p}, s}^{[1], \operatorname{adv}}(\mathbf{x}, t)\right)=0
$$

or, substituting the normal mode ansatz (9),

$$
0=e^{-i \omega t} \sum_{s=1}^{n} q^{(s)} \mathbf{c u r l} \operatorname{curl}\left[\sum_{\mathbf{p} \in \mathbb{Z}^{3}} e^{-i \mathbf{k} \cdot \mathbf{r}_{\mathbf{p}}}\left(\frac{e^{i \frac{\omega}{c}\left|\mathbf{x}-\mathbf{r}_{\mathbf{p}}^{(s)}\right|}}{\left|\mathbf{x}-\mathbf{r}_{\mathbf{p}}^{(s)}\right|}-\frac{e^{-i \frac{\omega}{c}\left|\mathbf{x}-\mathbf{r}_{\mathbf{P}}^{(s)}\right|}}{\left|\mathbf{x}-\mathbf{r}_{\mathbf{p}}^{(s)}\right|}\right) \mathbf{U}^{(s)}\right]
$$

This is immediately seen if one remarks the the only difference that shows up when considering the advanced fields occurs in the spherical-wave term, whose direction is inward rather than outward.

In order to prove the Wheeler-Feynman identity in the form (24), we rewrite the term in square brackets as a sum over the reciprocal lattice. Using the distributional identities (see e.g. [11])

$$
\begin{gathered}
\frac{e^{ \pm i \alpha x}}{x}=4 \pi \lim _{\epsilon \rightarrow 0^{+}} \int_{\mathbb{R}^{3}} d m(\mathbf{k}) \frac{e^{i \mathbf{k} \cdot \mathbf{x}}}{|\mathbf{k}|^{2}-\left(\alpha^{2} \pm i \epsilon\right)}, \\
\sum_{\mathbf{h} \in \mathbb{Z}^{3}} \delta\left(\mathbf{x}-\mathbf{r}_{\mathbf{h}}\right)=\frac{1}{\left|V_{c}\right|} \sum_{\mathbf{m} \in \mathbb{Z}^{3}} e^{i \mathbf{q}_{\mathbf{m}} \cdot \mathbf{x}},
\end{gathered}
$$

one gets

$$
\begin{aligned}
& \sum_{\mathbf{p} \in \mathbb{Z}^{3}} e^{-i \mathbf{k} \cdot \mathbf{r}_{\mathbf{p}}}\left(\frac{e^{i \frac{\omega}{c}\left|\mathbf{x}-\mathbf{r}_{\mathbf{p}}^{(s)}\right|}}{\left|\mathbf{x}-\mathbf{r}_{\mathbf{p}}^{(s)}\right|}-\frac{e^{-i \frac{\omega}{c}\left|\mathbf{x}-\mathbf{r}_{\mathbf{p}}^{(s)}\right|}}{\left|\mathbf{x}-\mathbf{r}_{\mathbf{p}}^{(s)}\right|}\right)= \\
& \quad \frac{4 \pi}{\left|V_{c}\right|} \sum_{\mathbf{m} \in \mathbb{Z}^{3}} \lim _{\epsilon \rightarrow 0^{+}}\left(\frac{e^{i\left(\mathbf{q}_{\mathbf{m}}-\mathbf{k}\right) \cdot\left(\mathbf{x}-\mathbf{b}^{(s)}\right)}}{\left|\mathbf{k}-\mathbf{q}_{\mathbf{m}}\right|^{2}-\left(\frac{\omega^{2}}{c^{2}}+i \epsilon\right)}-\frac{e^{i\left(\mathbf{q}_{\mathbf{m}}-\mathbf{k}\right) \cdot\left(\mathbf{x}-\mathbf{b}^{(s)}\right)}}{\left|\mathbf{k}-\mathbf{q}_{\mathbf{m}}\right|^{2}-\left(\frac{\omega^{2}}{c^{2}}-i \epsilon\right)}\right)=0 .
\end{aligned}
$$

So (24) is proven.

We finally show that the Wheeler-Feynman identity leads somewhat directly to Oseen identity, i.e., to the cancellation between the non-hermitian part of the dipole term matrix and the radiation reaction term in the linear equations of motion. 
To this end we let $\mathbf{x}$ approach a lattice site $\mathbf{r}_{\mathbf{h}}^{(j)}$, and rewrite (24) by separating the contribution of the $(\mathbf{h}, j)$ ion from that of all the other ones, thus getting

$$
\begin{aligned}
& 0=\frac{e^{-i \omega t}}{2} \operatorname{curl} \operatorname{curl}\left\{\sum_{(\mathbf{p}, s)}^{\prime}\left[q^{(s)} e^{i \mathbf{k} \cdot \mathbf{r}_{\mathbf{p}}}\left(\frac{e^{i \frac{\omega}{c}\left|\mathbf{x}-\mathbf{r}_{\mathbf{p}}^{(s)}\right|}}{\left|\mathbf{x}-\mathbf{r}_{\mathbf{p}}^{(s)}\right|}-\frac{e^{-i \frac{\omega}{c}\left|\mathbf{x}-\mathbf{r}_{\mathbf{p}}^{(s)}\right|}}{\left|\mathbf{x}-\mathbf{r}_{\mathbf{p}}^{(s)}\right|}\right) \mathbf{U}^{(s)}\right]+\right. \\
& \left.+\left[q^{(j)} e^{i \mathbf{k} \cdot \mathbf{r}_{\mathbf{h}}}\left(\frac{e^{i \frac{\omega}{c}\left|\mathbf{x}-\mathbf{r}_{\mathbf{h}}^{(j)}\right|}}{\left|\mathbf{x}-\mathbf{r}_{\mathbf{h}}^{(j)}\right|}-\frac{e^{-i \frac{\omega}{c}\left|\mathbf{x}-\mathbf{r}_{\mathbf{h}}^{(j)}\right|}}{\left|\mathbf{x}-\mathbf{r}_{\mathbf{h}}^{(j)}\right|}\right) \mathbf{U}^{(s)}\right]\right\} .
\end{aligned}
$$

A classical computation, first carried out by Dirac [19] (see also [20]), shows that the second term tends to the radiation reaction force. Since the first one is continuous at $\mathbf{r}_{\mathrm{h}}^{(j)}$, its limit must equal the opposite of the radiation reaction force. Now we observe that this quantity coincides with

$$
\frac{1}{q^{(j)}} e^{i\left(\mathbf{k} \cdot \mathbf{r}_{\mathbf{h}}-\omega t\right)} \sum_{s=1}^{n_{c}} \frac{1}{2}\left(\hat{\mathcal{D}}_{j s}-\hat{\mathcal{D}}_{s j}^{\dagger}\right) \cdot \mathbf{U}^{(s)} .
$$

To see this, factor $e^{i \mathbf{k} \cdot \mathbf{r}_{\mathbf{h}}}$ out of the sum, rename the summation index $\mathbf{p}-$ $\mathbf{h} \mapsto \mathbf{p}^{\prime}$ and compare the resulting expression with the corresponding term in (11). At last, it is not difficult to see that exchanging the indices $j$ and $s$ in the advanced field term yields the complex conjugate of the retarded one. This term amounts to one-half the difference between the retarded and the advanced fields generated by all ions but one, evaluated at the excluded ion site. When multiplied by the charge $q^{(j)}$, it becomes equal to the nonhermitian part of the dipole forces matrix. Therefore, the proof is complete: the unexpected cancellation appears now better justified from a theoretical point of view.

If one looks at the book of Born and Huang[1], one will see that, in dealing with the secular equation, they take into consideration only the real part of the equation, which is the one that actually produces the dispersion relation. Apparently they do not exploit the fact that the imaginary part identically vanishes if the contribution of the radiation reaction term is taken into account, and just altogether neglect the consideration of the imaginary part, as if did not exist. A reading of Born's book [9] of the year 1933 (see page 431) shows that the relevance of the classical radiation reaction force was well appreciated by him. However, he had to take into account the fact that it was not easy to fit such a force within quantum theory ${ }^{3}$.

\footnotetext{
${ }^{3}$ In the very words of Born: "Diese ganze klassische Theorie der Strahlungsdämpfung ist
} 


\section{B Proof of Ewald's formula}

In this appendix we prove Ewald's resummation formula, i.e., the formula which expresses the part of the field due to the "far" dipoles as a rapidly convergent series over the reciprocal lattice. Usually, in solid state physics Ewald's resummation formula is used in the static limit $\omega \rightarrow 0$, i.e., in order to resum the Coulomb fields of the far ions. We report here a proof of the full formula.

So, let us begin considering the following series

$$
\Psi(\mathbf{x}):=\sum_{\mathbf{p} \in \mathbb{Z}^{3}} e^{+i \mathbf{k} \cdot \mathbf{r}_{\mathbf{p}}} \frac{e^{i \frac{\omega}{c}\left|\mathbf{x}-\mathbf{r}_{\mathbf{p}}\right|}}{\left|\mathbf{x}-\mathbf{r}_{\mathbf{p}}\right|}
$$

which is the series that enters formula (17) of Section 5 for the electric field. Moreover, it enters also formula (10) of Section 3 for the dipole matrix $\hat{\mathcal{D}}_{j l}$, inasmuch as one has

$$
\begin{aligned}
\hat{\mathcal{D}}_{j l}=\left.q^{(j)} \sum_{\mathbf{p} \in \mathbb{Z}^{3}} q^{(l)} \hat{\mathcal{R}}\left(\frac{e^{-i \mathbf{k} \cdot \mathbf{r}_{\mathbf{p}}} e^{i \frac{\omega}{c}|\mathbf{x}|}}{|\mathbf{x}|}\right)\right|_{\mathbf{x}=\mathbf{r}_{\mathbf{p}}^{(j, l)}} & \\
& =\left.q^{(j)} q^{(l)} \hat{\mathcal{R}}\left(\Psi\left(\mathbf{x}+\mathbf{b}^{(j, l)}\right)\right)\right|_{\mathbf{x}=0}
\end{aligned}
$$

So, to prove both equation (15) of Section 3 and equation (18) of Section 5 , one needs to prove that

$$
\begin{aligned}
\Psi(\mathbf{x}) & =\frac{4 \pi}{\left|V_{c}\right|} \sum_{\mathbf{m} \in \mathbb{Z}^{3}} \frac{e^{-\frac{1}{4 \delta^{\prime 2}}\left(\left|\mathbf{q}_{\mathbf{m}}-\mathbf{k}\right|^{2}-\frac{\omega^{2}}{c^{2}}\right)}}{\left|\mathbf{q}_{\mathbf{m}}-\mathbf{k}\right|^{2}-\frac{\omega^{2}}{c^{2}}} e^{i\left(\mathbf{k}-\mathbf{q}_{\mathbf{m}}\right) \cdot \mathbf{x}} \\
& +\sum_{\mathbf{h} \in \mathbb{Z}^{3}}\left(e^{i \mathbf{k} \cdot \mathbf{r}_{\mathbf{h}}} \frac{2}{\sqrt{\pi}} \int_{\delta^{\prime}}^{+\infty} e^{\left(\frac{\omega^{2}}{4 c^{2}} \frac{1}{\eta^{2}}-\left|\mathbf{x}-\mathbf{r}_{\mathbf{h}}\right| \eta^{2}\right)} d \eta\right)
\end{aligned}
$$

with $\delta^{\prime}$ an arbitrary positive parameter.

To this end, we first reduce the series defining $\Psi(\mathbf{x})$ to a series over the

natürlich mit der heutigen Quantentheorie des Licht und der Materie nicht verträglich." 
reciprocal lattice, by using the identities already introduced in Appendix A

$$
\begin{gathered}
\frac{e^{ \pm i \alpha x}}{x}=4 \pi \lim _{\epsilon \rightarrow 0^{+}} \int_{\mathbb{R}^{3}} d^{3} k^{\prime} \frac{e^{i \mathbf{k}^{\prime} \cdot \mathbf{x}}}{\left|\mathbf{k}^{\prime}\right|^{2}-\left(\alpha^{2} \pm i \epsilon\right)}, \\
\frac{(2 \pi)^{3}}{\left|V_{c}\right|} \sum_{\mathbf{m} \in \mathbb{Z}^{3}} \delta\left(\mathbf{x}-\mathbf{q}_{\mathbf{m}}\right)=\sum_{\mathbf{p} \in \mathbb{Z}^{3}} e^{i \mathbf{r}_{\mathbf{p}} \cdot \mathbf{x}},
\end{gathered}
$$

$V_{c}$ being the cell volume, while $\mathbf{q}_{\mathbf{m}}$ are the vectors of the reciprocal lattice. We recall that, given a lattice of points $\mathbf{x}_{\mathbf{p}}$ in a vector space, the reciprocal lattice $\mathbf{k}_{\mathbf{m}}$ is the set of vectors of the dual space, namely, the vectors such that $\left\langle\mathbf{x}_{\mathbf{p}}, \mathbf{k}_{\mathbf{m}}\right\rangle$ is an integer multiple of $2 \pi$ (or zero). In $\mathbb{R}^{3}$, if $\mathbf{a}_{i}, i=1,2,3$, is a basis for the direct lattice, the vectors $\tilde{\mathbf{a}}_{k}=\left(2 \pi /\left|V_{c}\right|\right)\left(\mathbf{a}_{i} \wedge \mathbf{a}_{j}\right)$ constitute a basis for the reciprocal lattice. Using the mentioned identities one gets

$$
\begin{aligned}
\Psi(\mathbf{x}) & =\sum_{\mathbf{p} \in \mathbb{Z}^{3}} 4 \pi \lim _{\epsilon \rightarrow 0^{+}} \int_{\mathbb{R}^{3}} d^{3} k^{\prime} \frac{e^{i\left(\mathbf{k}-\mathbf{k}^{\prime}\right) \cdot \mathbf{r}_{\mathbf{p}}} e^{-i \mathbf{k}^{\prime} \cdot \mathbf{x}}}{|\mathbf{k}|^{2}-\left(\omega^{2} / c^{2}+i \epsilon\right)} \\
& =4 \pi \lim _{\epsilon \rightarrow 0^{+}} \int_{\mathbb{R}^{3}} d^{3} k^{\prime} \frac{e^{-i \mathbf{k}^{\prime} \cdot \mathbf{x}}}{|\mathbf{k}|^{2}-\left(\omega^{2} / c^{2}+i \epsilon\right)} \sum_{\mathbf{p} \in \mathbb{Z}^{3}} e^{i\left(\mathbf{k}-\mathbf{k}^{\prime}\right) \cdot \mathbf{r}_{\mathbf{p}}} \\
& =\frac{4 \pi}{\left|V_{c}\right|} \lim _{\epsilon \rightarrow 0^{+}} \int_{\mathbb{R}^{3}} d^{3} k^{\prime} \frac{e^{-i \mathbf{k}^{\prime} \cdot \mathbf{x}}}{|\mathbf{k}|^{2}-\left(\omega^{2} / c^{2} \pm i \epsilon\right)} \sum_{\mathbf{m} \in \mathbb{Z}^{3}} \delta\left(\mathbf{k}-\mathbf{k}^{\prime}-\mathbf{q}_{\mathbf{m}}\right) \\
& =\frac{4 \pi}{\left|V_{c}\right|} \sum_{\mathbf{m} \in \mathbb{Z}^{3}} \frac{e^{i\left(\mathbf{q}_{\mathbf{m}}-\mathbf{k}\right) \cdot \mathbf{x}}}{\left|\mathbf{k}-\mathbf{q}_{\mathbf{m}}\right|^{2}-\omega^{2} / c^{2}} .
\end{aligned}
$$

For any $\delta^{\prime}$, the series over the reciprocal lattice can be conveniently split as follows

$$
\begin{aligned}
\Psi(\mathbf{x})= & \Psi_{1}(\mathbf{x})+\Psi_{2}(\mathbf{x}):= \\
& \frac{4 \pi}{\left|V_{c}\right|} \sum_{\mathbf{m} \in \mathbb{Z}^{3}} \frac{e^{-\frac{1}{4 \delta^{\prime 2}}\left(\left|\mathbf{q}_{\mathbf{m}}-\mathbf{k}\right|^{2}-\frac{\omega^{2}}{c^{2}}\right)}}{\left|\mathbf{q}_{\mathbf{m}}-\mathbf{k}\right|^{2}-\frac{\omega^{2}}{c^{2}}} e^{i\left(\mathbf{k}-\mathbf{q}_{\mathbf{m}}\right) \cdot \mathbf{x}} \\
+ & \frac{4 \pi}{\left|V_{c}\right|} \sum_{\mathbf{m} \in \mathbb{Z}^{3}} \frac{1-e^{-\frac{1}{4 \delta^{\prime 2}}\left(\left|\mathbf{q}_{\mathbf{m}}-\mathbf{k}\right|^{2}-\frac{\omega^{2}}{c^{2}}\right)}}{\left|\mathbf{q}_{\mathbf{m}}-\mathbf{k}\right|^{2}-\frac{\omega^{2}}{c^{2}}} e^{i\left(\mathbf{k}-\mathbf{q}_{\mathbf{m}}\right) \cdot \mathbf{x}}
\end{aligned}
$$

where now the first series converges absolutely, while the second one can be expressed as an absolutely convergent series by going back to the direct 
lattice. In fact, using

$$
\frac{1-e^{-\frac{1}{4 \delta^{\prime 2}}\left(\left|\mathbf{q}_{\mathbf{m}}-\mathbf{k}\right|^{2}-\frac{\omega^{2}}{c^{2}}\right)}}{\left|\mathbf{q}_{\mathbf{m}}-\mathbf{k}\right|^{2}-\frac{\omega^{2}}{c^{2}}}=\int_{0}^{1 / 4 \delta^{\prime 2}} \mathrm{~d} \xi e^{-\xi\left(\left|\mathbf{q}_{\mathbf{m}}-\mathbf{k}\right|^{2}-\frac{\omega^{2}}{c^{2}}\right)}
$$

together with the identity (see below)

$$
\frac{(4 \pi \xi)^{3 / 2}}{\left|V_{c}\right|} \sum_{\mathbf{m} \in \mathbb{Z}^{3}} e^{-\left|\mathbf{q}_{\mathbf{m}}-\mathbf{k}\right|^{2} \xi} e^{i \mathbf{q}_{\mathbf{m}} \cdot \mathbf{x}}=\sum_{\mathbf{h} \in \mathbb{Z}^{3}} e^{-\frac{1}{4 \xi}\left|\mathbf{x}-\mathbf{r}_{\mathbf{h}}\right|^{2}} e^{i \mathbf{k} \cdot\left(\mathbf{x}-\mathbf{r}_{\mathbf{h}}\right)}
$$

one obtains

$$
\Psi_{2}(\mathbf{x})=\sum_{\mathbf{h} \in \mathbb{Z}^{3}} \int_{0}^{1 / 4 \delta^{\prime 2}} \mathrm{~d} \xi(4 \pi \xi)^{3 / 2} e^{-\frac{1}{4 \xi}\left|\mathbf{x}-\mathbf{r}_{\mathbf{h}}\right|^{2}} e^{i \mathbf{k} \cdot\left(\mathbf{x}-\mathbf{r}_{\mathbf{h}}\right)}
$$

and putting $\eta^{2}=1 / 4 \xi$ one gets

$$
\Psi_{2}(\mathbf{x})=\sum_{\mathbf{h} \in \mathbb{Z}^{3}}\left(e^{i \mathbf{k} \cdot \mathbf{r}_{\mathbf{h}}} \frac{2}{\sqrt{\pi}} \int_{\delta^{\prime}}^{+\infty} e^{\left(\frac{\omega^{2}}{4 c^{2}} \frac{1}{\eta^{2}}-\left|\mathbf{x}-\mathbf{r}_{\mathbf{h}}\right| \eta^{2}\right)} d \eta\right)
$$

which is the relation (26). To complete the proof, there remains to prove identity (28). This, however, is in fact rather straightforward, once one realizes that both sides of it are quasiperiodic functions of $\mathbf{x}$. One just has to show that the plane-wave coefficients at the left-hand side are indeed the Fourier coefficients of the function at the right-hand side, that we name $f(\mathbf{x})$ for convenience. Hence:

$$
\tilde{f}_{\mathbf{m}}=\int_{V_{c}} \frac{d^{3} y}{\left|V_{c}\right|} e^{-i \mathbf{q}_{\mathbf{m}} \cdot \mathbf{y}} f(\mathbf{y})=\frac{1}{\left|V_{c}\right|} \sum_{\mathbf{h} \in \mathbb{Z}^{3}} \int_{V_{c}} e^{-i \mathbf{q}_{\mathbf{m}} \cdot \mathbf{y}} e^{-\frac{1}{4 \xi}\left|\mathbf{y}-\mathbf{r}_{\mathbf{h}}\right|^{2}} e^{i \mathbf{k} \cdot\left(\mathbf{y}-\mathbf{r}_{\mathbf{h}}\right)} d^{3} y .
$$

We now apply the change of variable $\mathbf{y}^{\prime}=\mathbf{y}-\mathbf{r}_{\mathbf{h}}$, so that the integrand does no longer depend on $\mathbf{h}$ :

$$
\begin{aligned}
\widetilde{f}_{\mathbf{m}} & =\frac{1}{\left|V_{c}\right|} \sum_{\mathbf{h} \in \mathbb{Z}^{3}} \int_{V_{c}+\mathbf{r}_{\mathbf{h}}} e^{-\frac{1}{4 \xi}\left|\mathbf{y}^{\prime}\right|^{2}} e^{i\left(\mathbf{k}-\mathbf{q}_{\mathbf{m}}\right) \cdot \mathbf{y}^{\prime}} d^{3} y^{\prime} \\
& =\frac{1}{\left|V_{c}\right|} \int_{\mathbb{R}^{3}} e^{-\frac{1}{4 \xi}|\mathbf{y}|^{2}} e^{i\left(\mathbf{k}-\mathbf{q}_{\mathbf{m}}\right) \cdot \mathbf{y}} d^{3} y \\
& =\frac{1}{\left|V_{c}\right|} \prod_{\mu=1}^{3} \int_{\mathbb{R}} e^{-\frac{1}{4 \xi} y_{\mu}^{2}} e^{i\left(\mathbf{k}-\mathbf{q}_{\mathbf{m}}\right)_{\mu} y_{\mu}} d y_{\mu} .
\end{aligned}
$$


Next, we exploit the integral formula

$$
\int_{\mathbb{R}} e^{-\alpha x^{2}+\beta x} d x=\sqrt{\frac{\pi}{\alpha}} e^{\frac{\beta^{2}}{4 \alpha}}
$$

to finally get

$$
\tilde{f}_{\mathbf{m}}=\frac{(4 \pi \xi)^{3 / 2}}{\left|V_{c}\right|} e^{-\frac{1}{4 \xi}\left|\mathbf{k}-\mathbf{q}_{\mathbf{m}}\right|^{2}}
$$

Hence we have

$$
f(\mathbf{x})=\sum_{\mathbf{m} \in \mathbb{Z}^{3}} \tilde{f}_{\mathbf{m}} e^{i \mathbf{q}_{\mathbf{m}} \cdot \mathbf{x}}=\frac{(4 \pi \xi)^{\frac{3}{2}}}{\left|V_{c}\right|} \sum_{\mathbf{m} \in \mathbb{Z}^{3}} e^{-\frac{1}{4 \xi}\left|\mathbf{k}-\mathbf{q}_{\mathbf{m}}\right|^{2}} e^{i \mathbf{q}_{\mathbf{m}} \cdot \mathbf{x}},
$$

and the proof is complete.

\section{Computable form of the dynamical matrix}

For the sake of completeness, in this appendix we report the computable form of the dynamical matrix $\mathcal{A}(\mathbf{k}, \omega)$ that appears at the right-hand side of the generalized secular equation (11), i.e., after Ewald's summation has been performed.

The term $\mathcal{P}$ concerning the phenomenological repulsive short-distance interaction (first term at the right-hand side of (11)) is already in a computable form.

For the Coulomb term $\mathcal{C}$, we have

$$
\begin{aligned}
\frac{\hat{\mathcal{C}}_{j j}}{q^{(j)}} & =-\sum_{i \neq j} q^{(i)}\left\{\frac{4 \pi}{\left|V_{c}\right|} \sum_{\mathbf{m} \in \mathbb{Z}^{3}}^{\prime} \frac{e^{-\frac{|\mathbf{q} \mathbf{m}|^{2}}{4 \delta^{2}}}}{\left|\mathbf{q}_{\mathbf{m}}\right|^{2}} \hat{\mathcal{R}}\left[e^{i \mathbf{q}_{\mathbf{m}} \cdot \mathbf{x}}\right]_{\mathbf{x}=\mathbf{b}^{(j, i)}}+\sum_{\mathbf{h} \in \mathbb{Z}^{3}} \hat{\mathcal{R}}\left[\frac{\operatorname{erfc}(\delta|\mathbf{x}|)}{|\mathbf{x}|}\right]_{\mathbf{x}=\mathbf{r}_{\mathbf{h}}+\mathbf{b}^{(j, i)}}\right\} \\
& -q^{(j)}\left\{\frac{4 \pi}{\left|V_{c}\right|} \sum_{\mathbf{m} \in \mathbb{Z}^{3}}^{\prime} \frac{e^{-\frac{|\mathbf{q} \mathbf{m}|^{2}}{4 \delta^{2}}}}{\left|\mathbf{q}_{\mathbf{m}}\right|^{2}} \hat{\mathcal{R}}\left[e^{+i \mathbf{q}_{\mathbf{m}} \cdot \mathbf{x}}\right]_{\mathbf{x}=\mathbf{0}}+\sum_{\mathbf{h} \in \mathbb{Z}^{3}}^{\prime} \hat{\mathcal{R}}\left[\frac{\operatorname{erfc}(\delta|\mathbf{x}|)}{|\mathbf{x}|}\right]_{\mathbf{x}=\mathbf{r}_{\mathbf{h}}}+\hat{\mathcal{R}}\left[\frac{\operatorname{erf}(\delta|\mathbf{x}|)}{|\mathbf{x}|}\right]_{\mathbf{x}=\mathbf{0}}\right\} ;
\end{aligned}
$$

for all $j=1, \ldots, n$, and

$$
\hat{\mathcal{C}}_{j l}=0 \quad \text { if } j \neq l \text {. }
$$

The off-diagonal blocks vanish because the Coulomb term describes the force exerted on an ion by all the other charges, supposed fixed at their equilibrium positions: no coupling between two different ions occurs. 
Finally, for the dipole term $\mathcal{D}$, we have

$$
\begin{aligned}
\frac{\hat{\mathcal{D}}_{j j}}{\left(q^{(j)}\right)^{2}} & =\frac{4 \pi}{\left|V_{c}\right|} \sum_{\mathbf{m} \in \mathbb{Z}^{3}} \frac{e^{-\frac{1}{4 \delta^{\prime 2}}\left(\left|\mathbf{q}_{\mathbf{m}}-\mathbf{k}\right|^{2}-\frac{\omega^{2}}{c^{2}}\right)}}{\left|\mathbf{q}_{\mathbf{m}}-\mathbf{k}\right|^{2}-\frac{\omega^{2}}{c^{2}}} \hat{\mathcal{R}}\left[e^{i\left(\mathbf{q}_{\mathbf{m}}-\mathbf{k}\right) \cdot \mathbf{x}}\right]_{\mathbf{x}=\mathbf{0}} \\
& +\sum_{\mathbf{h} \in \mathbb{Z}^{3}}^{\prime} e^{-i \mathbf{k} \cdot \mathbf{r}_{\mathbf{h}}} \frac{2}{\sqrt{\pi}} \int_{\delta^{\prime}}^{+\infty} e^{\frac{\omega^{2}}{c^{2}} \frac{1}{\eta^{2}}} \hat{\mathcal{R}}\left[e^{-|\mathbf{x}|^{2} \eta^{2}}\right]_{\mathbf{x}=\mathbf{r}_{\mathbf{h}}} d \eta \\
& +\hat{\mathcal{R}}\left[\frac{1}{|\mathbf{x}|} \frac{2}{\sqrt{\pi}} \int_{\delta^{\prime}}^{\infty} e^{\frac{\omega^{2}}{4 c^{2}} \frac{1}{\eta^{2}}} e^{-|\mathbf{x}|^{2} \eta^{2}} d \eta-e^{i \frac{\omega}{c}|\mathbf{x}|}\right]_{\mathbf{x}=\mathbf{0}}
\end{aligned}
$$

for all $j=1, \ldots, n$, and

$$
\begin{aligned}
\frac{\hat{\mathcal{D}}_{j l}}{q^{(j)} q^{(l)}} & =\frac{4 \pi}{\left|V_{c}\right|} \sum_{\mathbf{m} \in \mathbb{Z}^{3}} \frac{e^{-\frac{1}{4 \delta^{\prime \prime 2}}\left(\left|\mathbf{q}_{\mathbf{m}}-\mathbf{k}\right|^{2}-\frac{\omega^{2}}{c^{2}}\right)}}{\left|\mathbf{q}_{\mathbf{m}}-\mathbf{k}\right|^{2}-\frac{\omega^{2}}{c^{2}}} \hat{\mathcal{R}}\left[e^{i\left(\mathbf{q}_{\mathbf{m}}-\mathbf{k}\right) \cdot \mathbf{x}}\right]_{\mathbf{x}=\mathbf{b}^{(j, l)}} \\
& +\sum_{\mathbf{h} \in \mathbb{Z}^{3}} e^{-i \mathbf{k} \cdot \mathbf{r}_{\mathbf{h}}} \frac{2}{\sqrt{\pi}} \int_{\delta^{\prime}}^{\infty} e^{\frac{\omega^{2}}{4 c^{2} \eta^{2}}} \hat{\mathcal{R}}\left[e^{-|\mathbf{x}|^{2} \eta^{2}}\right]_{\mathbf{x}=\mathbf{r}_{\mathbf{h}}^{(j, l)}} d \eta
\end{aligned}
$$

for all $j, l=1, \ldots, n, j \neq l$.

It can be seen that the total electric term (Coulomb plus dipole) takes the form of a standard "mechanical" dynamical matrix in the instantaneous limit, the potential being

$$
\phi_{(j, l)}(r)=\frac{q^{(j)} q^{(l)}}{r} .
$$

As already pointed out, this limit is formally obtained by putting $\omega=0$ in the matrix elements of $\mathcal{D}$. Thereby, the electric force matrix $\mathcal{C}+\mathcal{D}$ (actually its hermitian part) reduces to a "mechanical" dynamical matrix, analogous to $\mathcal{P}$, the only difference being related to the long range of the interaction. However, the purpose of this work is to investigate precisely those situations where the instantaneous limit loses validity.

\section{References}

[1] M. Born, K. Huang, Dynamical theory of crystal lattices, (Oxford University Press, Oxford, 1954) 91.

[2] U. Fano, Phys. Rev. 103, (1956) 1202. 
[3] J.J. Hopfield, Phys. Rev. 112, (1958) 1555.

[4] G. Grosso, G. Pastori Parravicini, Solid State Physics, (Academic Press, San Diego and London, 2000) 239.

[5] J.A. Wheeler, R.P. Feynman, Rev. Mod. Phys. 17, (1945) 157.

[6] J.D. Jackson, Classical electrodynamics, (J. Wiley and Sons, New York and London, 1998).

[7] P.P. Ewald, Ann. d. Phys 54, (1917) 519; 64, 253 (1917).

[8] C.W. Oseen, Physik. Z. 17, (1916) 341.

[9] M. Born, Optik, (Springer, Berlin, 1933) 431.

[10] M. Born, E. Wolf, Principles of optics, (Pergamon Press, Oxford, 1959) 79.

[11] A.N. Tikhonov, A.A. Samarskij, Equations of Mathematical Physics, (Pergamon press, Oxford 1963) 491.

[12] P. Gibbon, G. Sutmann, in Quantum Simulation of Complex Many-Body Systems: from Theory to Algorithms, J. Grotendorst, D. Marx, A. Muramatsu eds., NIC Series 10, (John von Neumann Institute for Computing, Jülich, 2002) 467.

[13] A. Carati, F. Benfenati. A. Maiocchi, M. Zuin, L. Galgani, Chaos (2013), in press.

[14] G. Dolling, H.G. Smith, R.M. Nicklow, P.R. Vijayaraghavan, M.K. Wilkinson, Phys. Rev. 168, (1968) 970.

[15] A. Carati, L. Galgani, Nuovo Cim. B 118, (2003) 839.

[16] M. Marino, A. Carati, L. Galgani, Ann. Phys. 322, (2007) 799.

[17] K. Hisano, Y. Okamoto, O. Matumura, J. Phys. Soc. Japan 28, (1970) 2.

[18] M. Born, Problems of atomic dynamics, (Dover, New York, 2004) 163.

[19] P.A.M. Dirac, Proc. Royal Soc. (London) A 167, (1938) 148.

[20] M. Marino, Ann. Phys. 301, (2002) 85. 